\title{
E-BIACS: Um Sistema para a Construção de Ambientes Virtuais para Aprendizagem Baseada em Problemas
}

\author{
Marcos Feier Fróes - UFRGS - marcos.froes@ufrgs.br \\ Eduardo Ribas Santos - UFRGS - ersantos@ea.ufrgs.br
}

Resumo: No desenvolvimento de ambientes virtuais para aprendizagem, é fundamental levar em conta a abordagem pedagógica. Neste artigo apresentamos um sistema para a construção de ambientes virtuais para aprendizagem, onde princípios construtivistas são desenvolvidos a partir de uma abordagem baseada em problemas. O sistema foi aplicado no domínio de gestão de projetos e o ambiente construído foi avaliado por gerentes de projetos. Na avaliação dos participantes, o ambiente proporcionou atividades autênticas, as ações foram organizadas de forma a despertar a curiosidade, os recursos instrucionais recomendados estavam contextualizados com as situações problemáticas e foram considerados adequados no processo de elucição dos problemas. Palavras-chaves: aprendizagem baseada em problemas, ambientes complexos de aprendizagem, sistemas de recomendação.

\section{E-BIACS: A System to Construct Virtual Problem-Based Learning Environments}

Abstract: In order to construct a virtual learning environment is fundamental to consider the pedagogical approach. A system to construct virtual learning environments where constructivist principles are developed from a problem-based approach is presented. The system was applied to the project management domain and the environment was evaluated by a group of project managers. According to the participants, the activities were authentic, the actions were organized to stimulate curiosity, and the recommended instructional resources were related to the problems and useful to elucidate them.

Keywords: problem-based learning; complex teaching-learning environments, recommender systems.

\section{INTRODUÇÃO}

Este projeto é parte das iniciativas de um grupo de pesquisa de uma Universidade brasileira, buscando a aplicação de tecnologias para a diversificação das abordagens pedagógicas no ensino superior em Administração no Brasil. Para o desenvolvimento de ambientes virtuais para aprendizagem em Administração, os chamados ambientes complexos de aprendizagem (Pellegrino, 2004) (Achtenhagen, 2001) demonstram-se especialmente adequados, compreendendo ambientes compatíveis com princípios construtivistas (Mondadori e Santos, 2006). Segundo Seel e Dijkstra (2004), no desenvolvimento de um ambiente para aprendizagem, é fundamental que se leve em conta a abordagem pedagógica. A Aprendizagem Baseada em Problemas (Problem-Based Learning, ou PBL) é um modelo pedagógico em grande sintonia com princípios construtivistas (Gijbels et al., 2006). A PBL se distingue por fazer de um problema o ponto de partida para o processo de aprendizagem, e desde sua origem tem sido aplicada em numerosos programas de diversos domínios tais como Medicina, Direito, Economia, Administração e Ciências Sociais (Loyens et al., 2008). Através dos problemas, procura-se estimular a curiosidade do aprendiz a fim de motivá-lo a buscar o conhecimento. É preciso, em contrapartida, ter-se disponíveis os recursos instrucionais 
adequados e suficientes para instruir o aprendiz sobre os aspectos fundamentais do problema. Neste contexto, mecanismos de recomendação ganham relevância.

A idéia de mecanismos de recomendação já é tema de relevantes pesquisas (López-Nores et. al., 2012). Em se tratando de um contexto de aprendizagem, a recomendação de recursos instrucionais deve levar em conta as estratégias pedagógicas do professor e ter como referência os conhecimentos do domínio. Segundo Adda et. al. (2007), ontologias constituem a forma padrão de representação dos conceitos e relações de um domínio. Diversas pesquisas utilizaram ontologias no contexto de recomendações em sistemas (Ferreira-Satler et. al., 2012) (Liao et. al., 2009) (Adda et. al., 2007). No contexto dos ambientes complexos de aprendizagem, o conhecimento sobre o domínio representado em uma ontologia corresponderia a um "modelo da realidade" tal como definido por Achtenhagen (2001).

Este artigo apresenta o E-BIACS, um sistema que possibilita a construção de ambientes virtuais para aprendizagem baseada em problemas. Os ambientes para aprendizagem no E-BIACS são formados por conjuntos de situações problemáticas, onde as questões relacionadas aos objetivos de aprendizagem são enunciadas a partir de problemas realísticos. Junto às situações problemáticas, o ambiente recomenda um conjunto de recursos instrucionais contextualizados com o problema. O sistema foi aplicado na construção de um ambiente para aprendizagem em gestão de projetos e o ambiente foi avaliado por um grupo de gerentes de projetos.

\section{REFERENCIAL TEÓRICO}

\subsection{Aprendizagem Baseada em Problemas}

A metodologia PBL foi introduzida inicialmente na Universidade de McMaster, Canadá, que a implantou na Faculdade de Ciências Médicas em 1969 (Sales et. al., 2013). A idéia era proporcionar aos estudantes de medicina, desde o início do curso, uma prática (virtual) do trabalho com pacientes reais. Apesar de muitas variações terem sido desenvolvidas, Barrows (1996) descreve um modelo central de PBL a partir de seis características (Gijbels et al., 2006): a aprendizagem deve ser centrada nos estudantes; a aprendizagem deve ocorrer em pequenos grupos de estudantes sob a condução de um tutor; o tutor é um facilitador ou guia; problemas autênticos são enfrentados antes de ocorrer qualquer preparação ou estudo; os problemas são utilizados como uma ferramenta para atingir o conhecimento desejado e as habilidades de resolução de problemas necessárias para eventualmente resolvê-los; e novas informações são adquiridas através do aprendizado "auto dirigido".

Segundo Sherwood (2004), a aprendizagem baseada em problemas tem um grande potencial para aprendizagem em Administração. Para o autor, colocar os aprendizes em um ambiente centrado em problemas pode ajudar a criar uma ponte entre a teoria e a prática. Kloppenborg e Baucus (2004) apresentaram uma experiência de utilização da PBL para aprendizagem em gestão de projetos na Universidade Xavier, EUA. Através da parceria com organizações sem fins lucrativos, os alunos têm a oportunidade de trabalhar com projetos reais e, em contrapartida, contribuir com essas organizações. Segundo os autores, esta abordagem prática se torna mais desafiante aos alunos, pois eles precisam lidar com complexidades maiores do que na maioria dos casos utilizados nos cursos de Administração de Projetos tradicionais.

\subsection{Ambientes Complexos de Aprendizagem}

O termo ambientes complexos de aprendizagem é derivado de Pellegrino (2004) e Achtenhagen (2001) para designar ambientes virtuais de aprendizagem compatíveis com princípios construtivistas (Mondadori e Santos, 2006). Os ambientes complexos de 
aprendizagem têm suas origens nos campos da psicologia cognitiva e do design instrucional. Têm em comum a organização de temas de aprendizado inter-relacionados através de um problema a ser resolvido ou meta a ser atingida, o emprego de tecnologia multimídia e o foco no aprendiz (Pellegrino, 2004) (Achtenhagen, 2001).

Achtenhagen (2001) elenca diversos critérios para o desenvolvimento destes ambientes: os aprendizes devem enfrentar experiências de problemas e fatos relativamente complexos que possam ser relacionados à realidade; o ensino deve levar em conta os conhecimentos e interesses dos aprendizes; deve iniciar por uma meta complexa, que possa servir como um guia para os tópicos a serem ensinados; o conhecimento deve ser desenvolvido resolvendo-se problemas que possuam significado; os objetos de aprendizagem devem ser acessíveis e abertos à experimentação; o conflito entre processos relacionados a casos e processos sistemáticos deve ser equilibrado, utilizando-se uma perspectiva sistêmica orientada à ação; o ensino deve ensejar uma perspectiva metacognitiva: aprender "sobre o modelo"; devem ser oferecidos problemas não estruturados, que não sejam facilmente solucionáveis por um trabalho de equipe meramente "aditivo"; o ambiente deve conter desafios semelhantes aos encontrados no ambiente de trabalho, propiciando transferibilidade e mobilidade do conhecimento. Segundo o autor, o projeto de um ambiente envolve quatro etapas:

1. especificação dos objetivos pedagógicos,

2. definição das estratégias instrucionais,

3. produção das tarefas de exploração (que se referem ao processo de navegação que o estudante deve vivenciar na busca da resolução de um problema complexo, coletando e estruturando os dados necessários),

4. construção de um modelo (Modelo1) contendo os aspectos de interesse da realidade e de um modelo sob a perspectiva didática (Modelo2).

A Figura 1 ilustra a abordagem proposta por Achtenhagen (2001).

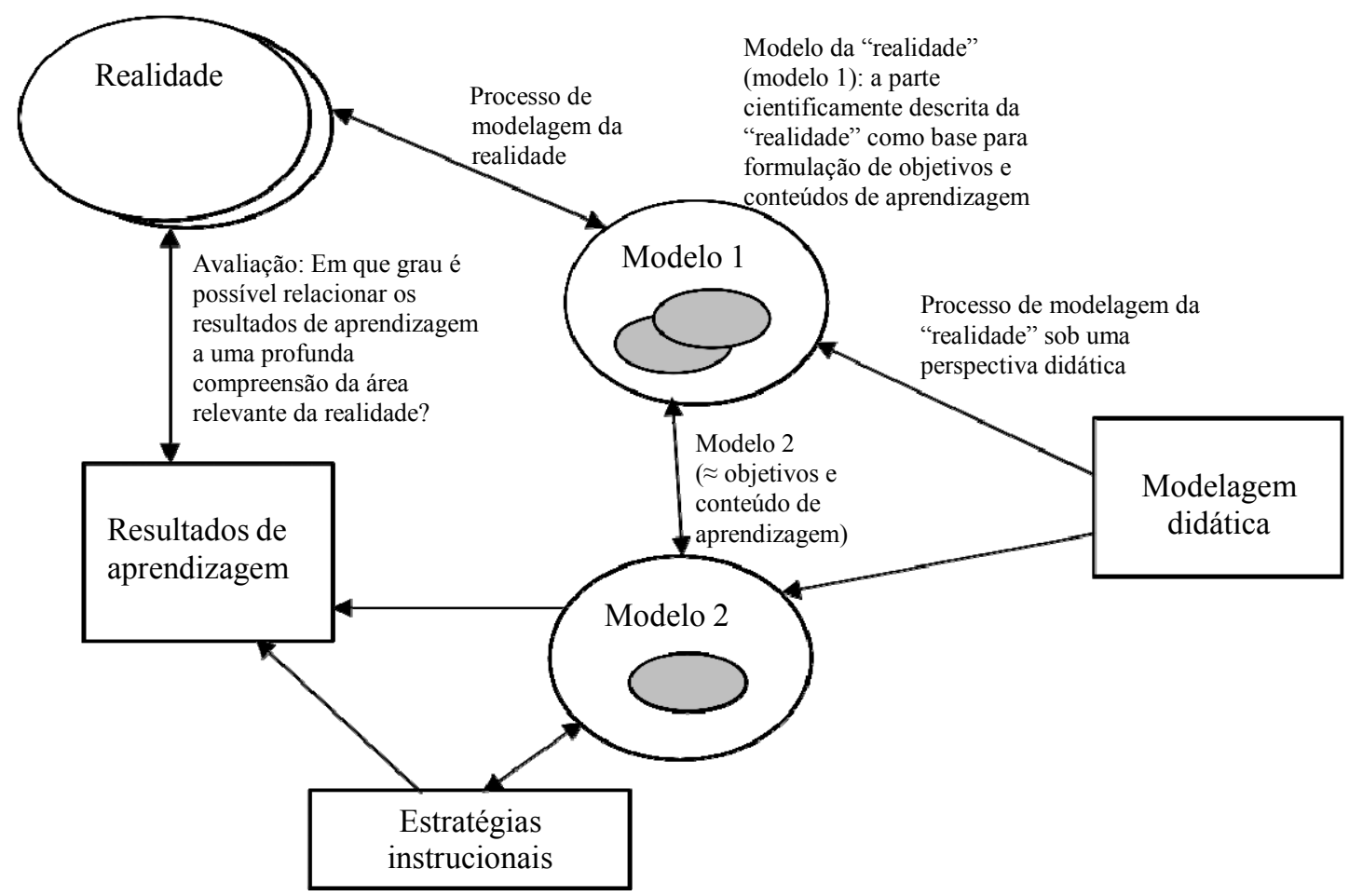

Figura 1. Processo de modelagem da realidade para propósitos instrucionais. (Achtenhagen, 2001) 


\subsection{Sistemas de Recomendação}

Os sistemas de recomendação empregam técnicas de filtragem de informação e de aprendizagem de máquina para gerar recomendações apropriadas aos usuários (Menezes et. al., 2013). Conforme ao modo como as recomendações são realizadas, os sistemas de recomendação encontram-se, usualmente, classificados em (Maidel et. al., 2010):

- Recomendações baseadas no conteúdo: ao usuário serão recomendados itens similares àqueles que o mesmo mostrou preferência em ocasiões anteriores;

- Recomendações colaborativas: itens desejados no passado por pessoas com gostos e preferências similares aos do usuário, serão recomendados;

- Abordagens híbridas: métodos que combinam as duas categorias.

Um passo à frente em relação à caracterização de qualidades e diversidade do usuário compreende a aplicação de técnicas da web semântica na recomendação (López-Nores et. al., 2012). Esta abordagem, através de processos automatizados de raciocínio, elucida as questões em jogo a partir de uma recuperação do significado dos termos empregados pelo usuário. Por exemplo, a partir de dois termos, o nome de duas raças de cachorro, é possível estabelecer a qual categoria pertencem: raça de cachorro. Este processo se dá perante o emprego de uma ontologia (Kanellopoulos et. al., 2006), onde uma representação do conhecimento proporciona a recuperação do significado que uma coleção de termos possa assumir em determinadas condições bem estabelecidas.

Liao et. al. (2009) apresentaram um modelo ontológico para personalização de serviços de recomendação em bibliotecas digitais. Ferreira-Satler et. al. (2012) demonstraram como uma ontologia fuzzy pode ser utilizada para representar perfis de usuários dentro de um mecanismo de recomendação para melhorar as atividades dos alunos, tutores e professores em um ambiente de e-learning.

\section{E-BIACS: UM SISTEMA PARA CONSTRUÇÃO DE AMBIENTES VIRTUAIS PARA APRENDIZAGEM BASEADA EM PROBLEMAS}

A abordagem adotada para a concepção do E-BIACS parte da idéia de integrar as proposições de Achtenhagen (2001) e a PBL. Sendo assim, da integração do modelo de Achtenhagen que contém a definição dos objetivos e conteúdos de aprendizagem com a abordagem da aprendizagem baseada em problemas, pode ser derivado outro modelo que contém um conjunto de situações problemáticas, onde as questões relacionadas aos objetivos de aprendizagem estejam enunciadas a partir de problemas. Para dar suporte às decisões do aprendiz na sua lide com cada questão relacionada ao problema, deve estar acessível um conjunto de recursos instrucionais, adequadamente contextualizados. Neste ponto, um elemento que faça esta ponte (entre os recursos instrucionais e as atitudes do aprendiz perante os problemas) é necessário. Conforme Achtenhagen, embora não explicite como, esta ponte é viabilizada através de um modelo que contenha a parte cientificamente descrita da "realidade". Semelhante ao proposto por Pereira e Rigo (2013), a solução adotada para o E-BIACS compreende o emprego de uma ontologia de domínio. Nesta ontologia são representados os fenômenos relacionados ao domínio dos conhecimentos (formais) que, teoricamente descrevem fenômenos subjascentes ao que se passa nas situações problemáticas. Através de tal ontologia, que será denominada de "modelo de conhecimentos", é possível:

- mapear um conjunto de cenários fenomenológicos à cada situação problemática;

- indexar os recursos instrucionais pertinentes.

Os modelos de conhecimentos do E-BIACS tomam por base a abordagem utilizada no sistema "BIACS - Base Inteligente para Aquisição de Conhecimentos de 
Sistemas" (Santos, 1989). Esta abordagem foi adotada por ser flexível, permitindo representar conhecimentos em diferentes níveis de complexidade. Os modelos procuram descrever fenômenos de um domínio seguindo os princípios do pensamento sistêmico (Checkland, 1981). Assim, pode-se definir: os sistemas e subsistemas, as variáveis que caracterizam o estado de um sistema e as condições em que os estados desejados são esperados. Também se definem os parâmetros (requisitos de design) do sistema, as alterações possíveis em relação ao desejado para cada estado e as alterações possíveis em relação a seus requisitos de design para cada parâmetro. Um sistema é definido a partir do estabelecimento de suas fronteiras pelo criador do modelo. As fronteiras de um sistema são convencionadas, de maneira a acomodar da maneira mais adequada as relações entre as variáveis de estado que caracterizam os fenômenos que se quer representar.

No modelo de conhecimentos também são definidas relações entre os sistemas e subsistemas através de associações de causa-conseqüência entre as alterações de estados desejados e as deteriorações de requisitos de design dos sistemas. Proposições causais incluem um conjunto de condições e um conjunto de efeitos conectados por uma relação causal (Jonassen e Ionas, 2008). O conjunto destas relações compreende um grafo orientado, onde redes causais hipotéticas são formadas dinamicamente. Cada rede causal descreve um comportamento do sistema em uma condição bem estabelecida, onde os fenômenos presentes são abordados em vários níveis de abstração.

O processo para a modelagem de conhecimentos compreende as seguintes etapas:

1. Uma representação hierárquica do sistema. Nesta, cada subsistema constitui um nível de abstração do problema.

2. A representação das características de cada subsistema em estado "normal" ou desejado na concepção do sistema. Cada subsistema é caracterizado através de suas variáveis de estado, descrevendo comportamentos normais. Também são representados sinais através dos quais um estado do sistema pode ser avaliado.

3. A representação da deterioração de um estado do sistema, descrevendo desvios de comportamento. Também são representados sintomas, através dos quais a deterioração de um estado do sistema pode ser avaliada.

4. Associações entre desvios de comportamento e entre desvios de comportamento e sintomas, que descrevem o problema em níveis de abstração.

5. A representação das características de design do sistema, descritas através de parâmetros de projeto.

6. A representação de não-conformidades em relação às características de design adequadas ao sistema, descritas através de falhas.

7. Associações entre falhas e desvios de comportamento.

$\mathrm{O}$ processo de modelagem é realizado em dois sentidos. Em um sentido representam-se e, em outro, identificam-se subsistemas. A identificação de (novos) subsistemas dá-se em função da resolução de falhas elucidadas, e da sua associação com funcionalidades já representadas no modelo. Ao final deste processo, tem-se no EBIACS o equivalente ao Modelo1 descrito por Achtenhagen (2001).

A construção do Modelo 2 de Achtenhagen (2001), que introduz os objetivos e o conteúdo de aprendizagem, se dá em duas etapas: a seleção e organização dos recursos instrucionais conforme ao modelo de conhecimentos e a modelagem das situações problemáticas para o ambiente virtual de aprendizagem. No E-BIACS, uma situação problemática é definida a partir de um script que descreve parte de um caso realístico. Pode ser apresentada ao aprendiz como um texto, um filme, ou outro meio considerado apropriado pelo professor. A cada um destes scripts pode estar associado um conjunto 
de documentos (documentos técnicos, atas de reuniões, etc) que ajudam a contextualizar o problema. A cada situação problemática deve corresponder um ou mais desvios de comportamento ou falhas que contextualizam o problema a partir do modelo de conhecimentos. Esta configuração será o ponto de partida para a recomendação dos recursos instrucionais. Os recursos instrucionais são recomendados em quantidade configurável pelo professor, de acordo com a proximidade na estrutura das redes causais instanciadas a partir de algoritmos de simulação (dado o sintoma, buscar as consequências) e diagnóstico (dado o sintoma, buscar as causas), partindo dos desvios de comportamento ou falhas configurados.

\section{UM AMBIENTE PARA APRENDIZAGEM EM GESTÃO DE PROJETOS}

$O$ primeiro passo para a criação de um ambiente para aprendizagem em gestão de projetos foi a construção de um modelo de conhecimentos do domínio. O modelo foi construído com base na experiência de um professor da disciplina de Administração de Projetos. Na Figura 2 estão apresentados os sistemas e subsistemas modelados pelo professor, cuja elucidação teve origem na idéia de que cada coluna do software de gestão de projetos utilizado nas aulas práticas poderia ser relacionada a um sistema nas atividades de gestão de um projeto. Por exemplo, o sistema para definição das atividades contém, efetivamente, os processos para a construção da estrutura analítica do projeto, cujas atividades têm como atributos os demais dados para a gestão.

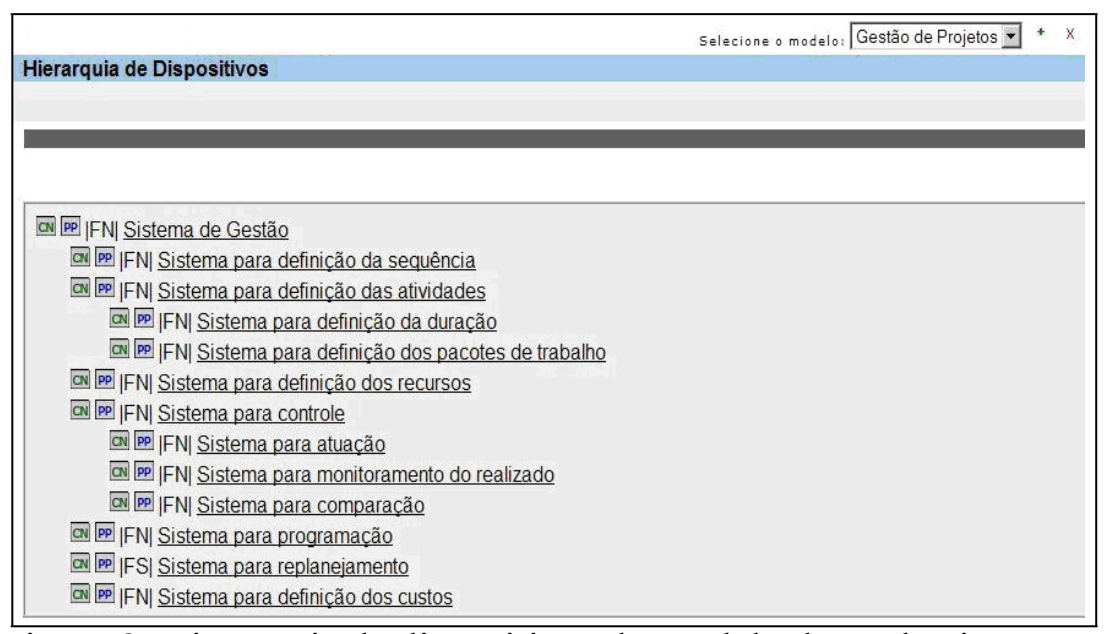

Figura 2. Hierarquia de dispositivos do modelo de conhecimentos.
A duração, as atividades

predecessoras, os recursos, etc. são atributos de uma atividade. Logo, os processos para a sua definição também podem ser representados a partir da definição de outros sistemas para a gestão de um projeto.

A Figura 3 ilustra a representação de estados desejados do sistema para replanejamento, contendo, a definição de sob qual condição o estado é possível. Duas condições estão representadas: a primeira situa o comportamento do sistema para replanejamento na sua condição usual, que é a de fornecer o ferramental gerencial para ajustes no cronograma durante a execução do projeto; a segunda descreve o comportamento esperado do sistema em uma condição de emergência, quando houve uma perda definitiva no prazo do projeto: o prazo, neste caso, deverá ser negociado junto ao cliente.

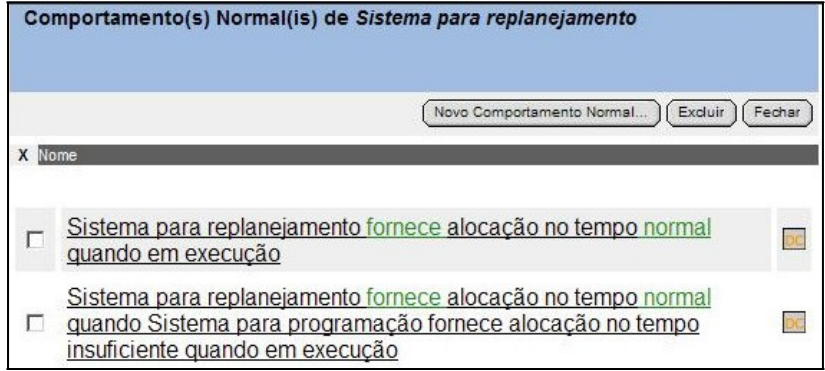

Figura 3. Estados desejados do sistema para replanejamento.

Um cenário de pânico está definido na Figura 4, quando mesmo após uma 
renegociação do prazo, as coisas não deram certo. A Figura 5 refere-se à geração

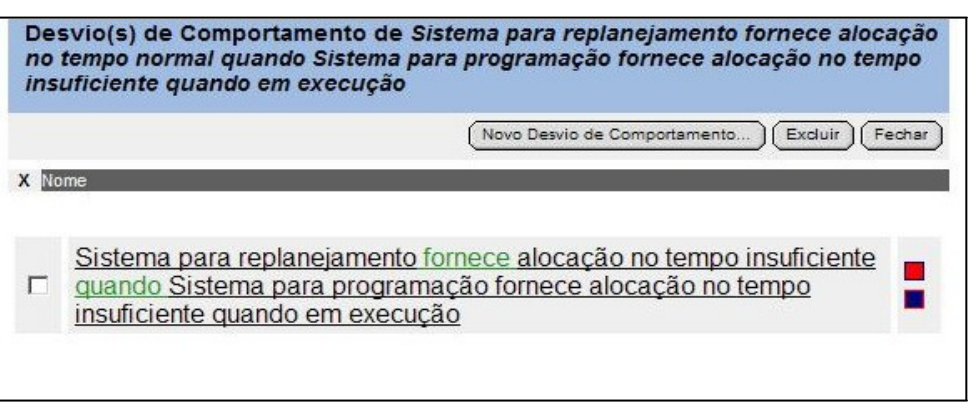

Figura 4. Alteração de estado desejado do sistema para replanejamento. dinâmica de redes causais. Estas redes são geradas a partir de diagnósticos e de simulações de uma alteração de estado desejado ou de uma deterioração de requisito de design de um sistema do modelo de conhecimentos.

Cada rede, portanto, compreende um cenário problemático.

Concluído o
modelo, partiu-se para
a seleção e indexação
dos recursos
instrucionais. Ao todo,
foram 54 recursos
indexados entre livros,
artigos, sites, vídeos e
imagens. O criterio
para associar um
recurso instrucional a
um elemento do
modelo partiu de uma avaliação individualizada, observando a ênfase e a profundidade que o material trata sobre cada tema relevante para um determinado contexto. Por exemplo, à alteração de estado desejado sistema para definição da sequência fornece vértices de um grafo orientado inadequados quando em planejamento foram associados recursos instrucionais com maior ênfase em assunto como redes PERT e método do caminho crítico, pela relação desses assuntos com o planejamento da sequência das atividades.

A modelagem das situações problemáticas partiu da seleção de um projeto autêntico em uma organização real e prosseguiu com a coleta de informações e documentos através de reuniões com envolvidos no projeto. $\mathrm{O}$ caso autêntico utilizado foi o projeto do portal internet da rádio de uma Assembleia Legislativa estadual brasileira. O projeto foi desenvolvido pelo departamento de tecnologia da organização, que subsidiou esta pesquisa com documentos sobre o projeto. O objetivo pedagógico do ambiente partiu da idéia de estimular o entendimento geral sobre a disciplina de gestão de projetos e, em especial, sobre a estrutura analítica de projeto (EAP). O ambiente, inicialmente, apresenta a situação problemática "a solicitação do cronograma do projeto do Portal da Rádio Assembleia”. A situação problemática inicial (Figura 6) foi inspirada em relatos de que houve pressão sobre os prazos no projeto real. $\mathrm{O}$ aprendiz é colocado na posição de quem acaba de assumir o projeto e precisa elaborar o cronograma. A principal situação problemática do ambiente instiga à definição das atividades do projeto. Perspectivas sobre estimativas para a duração das atividades e mapeamento de dependências entre atividades foram incluídas, pela relação com a programação do 
cronograma. Os documentos coletados (atas de reuniões, memorandos, etc) foram anexados às situações problemáticas conforme o assunto e o conteúdo. Cada situação problemática foi configurada a um contexto problemático (causa ou consequência) no modelo de conhecimentos. Por exemplo, a situação problemática inicial, relacionada ao cronograma do projeto, foi associada à alteração de estado desejado sistema para programação fornece alocação no tempo insuficiente quando em planejamento, por ser uma falha comum na programação de um cronograma em um contexto de pressão por prazo a previsão insuficiente de tempo.

\begin{tabular}{|c|c|}
\hline A solicitação do cronograma do Portal da Rádio Assembleia & Recursos Recomendados: \\
\hline \multirow{4}{*}{$\begin{array}{l}\text { O Departamento de Jornalismo da Superintendência de Comunicação Social encaminhou ao } \\
\text { Departamento de Tecnologia da Informação (DTI, antigo DSI) a solicitação para o } \\
\text { desenvolvimento de um um novo portal para a Rádio da Assembleia. Esta solicitação foi o sinal } \\
\text { verde para a execução de um projeto já discutido em algumas oportunidades pelas áreas } \\
\text { envolvidas e apresentado pelo DTI ao Superintendente de Comunicação Social. } \\
\text { Você foi recentemente designado para a função de Coordenador da Divisão de Internet, Intranet e } \\
\text { Portais e, por isso, o Diretor do DTI solicitou-lhe a elaboração do cronograma do projeto, que será } \\
\text { de sua responsabilidade. A informação sobre a data final do projeto é aguardada, com certa } \\
\text { ansiedade, para que possa ser finalizado o material de mídia a ser amplamente divulgado. Os } \\
\text { superintendentes estão preocupados com o prazo do projeto em relação às demais iniciativas do } \\
\text { plano de melhorias da área de comunicação social da instituição e pressionaram o diretor do DTI } \\
\text { para que os prazos não se alonguem e sejam rigorosamente cumpridos. }\end{array}$} & $\begin{array}{l}\text { Esquema resumido dos processos de Gestão de projetos. } \\
\text { PMTECH : Visão macro das etapas de um projeto. }\end{array}$ \\
\hline & $\begin{array}{l}\text { Fluxo resumido de processos do gerenciamento de } \\
\text { projetos - PMBOK 4. PMTECH: Fluxograma dos processos } \\
\text { do gerencimamento de projetos. }\end{array}$ \\
\hline & $\begin{array}{l}\text { Visão geral dos processos do gerenciamento de projetos - } \\
\text { PMBBOK 4. PMTECH: Esquema gráfico dos processos de } \\
\text { gerenciamento de projetos de acordo com o PMBOK. }\end{array}$ \\
\hline & $\begin{array}{l}\text { Vídeoaula. Gerência de Tempo: Desenvolvimento do } \\
\text { Cronograma (Teoria): Filmagem de uma aula sobre } \\
\text { aspectos relacionados ao desenvolvimento do cronograma } \\
\text { de um projeto. }\end{array}$ \\
\hline $\begin{array}{l}\text { Pesquisando no diretório compartilhado de projetos, você encontrou uma pasta com nome } \\
\text { "Projeto Portal da Rádio Web" com os seguintes documentos: }\end{array}$ & $\begin{array}{l}\text { Vídeoaula. Gerência de Tempo. Desenvolvimento do } \\
\text { Cronograma (Prática): Filmagem de uma aula sobre } \\
\text { aspectos práticos relacionados ao desenvolvimento do } \\
\text { cronograma de um projeto. }\end{array}$ \\
\hline $\begin{array}{l}\text { 27/03/2008 Ata de reunlao sobre a area do site que diz respelto a Radio web } \\
\text { 25/03/2009 Parecer dos analistas da Divisão de Portais }\end{array}$ & $\begin{array}{l}\text { [www] PMBOK: Desenvolvimento do Cronograma: } \\
\text { Hyperdocumento hospedado nos servidores da UFPE que } \\
\text { contém informaçōes sobre Gerenciamento do tempo. }\end{array}$ \\
\hline $\begin{array}{l}\text { *30/03/2009 Modelos suqeridos pela Comunicação Social para o novo portal } \\
\text { * 30/03/2009 Esbocco para interface do novo portal }\end{array}$ & $\begin{array}{l}\text { Gerência do tempo do projeto. Tradução livre do PMBBOK V } \\
\text { 1.0. PAMI MG : Tradução do capitulo } 6 \text { do PMBOK sobre } \\
\text { Gerência do Tempo do Projeto. } 15 \text { páginas. }\end{array}$ \\
\hline \multicolumn{2}{|l|}{ Ir para: } \\
\hline \multicolumn{2}{|l|}{ A conversa com os analistas da Divisão de Portais para a definição das atividades do projeto } \\
\hline A reunião com Diretor do DTI para a definição dos recursos do projeto & \\
\hline
\end{tabular}

Figura 6. Ambiente de aprendizagem no E-BIACS: Situação problemática relacionada à definição do cronograma de um projeto.

\section{AVALIAÇÃO E DISCUSSÕES}

Sete gerentes de projetos foram voluntários para participar de um experimento de avaliação do ambiente. Após navegarem livremente, os participantes responderam a um questionário (Fróes e Santos, 2014). Todos os participantes concordaram com a afirmação o sistema proporcionou atividades autênticas. Proporcionar atividades autênticas está entre os principais objetivos no design de um ambiente complexo de aprendizagem (Mondadori e Santos, 2006). Também foi unânime a concordância com a afirmação as ações no sistema estão organizadas de forma a despertar a curiosidade. Trata-se de um indício de que o objetivo de estimular a curiosidade dos aprendizes foi atingido na modelagem do ambiente. Acredita-se que esta curiosidade ajude a criar condições para o aprendizado "auto dirigido", tão defendido pelos autores em PBL (Loyens et. al., 2008). Também foi geral a concordância com as afirmações de que os recursos instrucionais recomendados pelo sistema estavam contextualizados com a situação problemática e os recursos instrucionais recomendados pelo sistema foram úteis no processo de elucidar a situação problemática. Isso indica que a seleção e a organização dos recursos instrucionais foram adequadas e que o mecanismo de recomendação apresentou funcionamento satisfatório. 


\section{CONSIDERAÇÕES FINAIS}

Mesmo que não se tenha uma avaliação mais profunda a respeito dos resultados de aprendizagem dos ambientes construídos no E-BIACS e de como seria a sua utilização em programas regulares, as percepções manifestadas pelos participantes da pesquisa foram bastante positivas. Isso pode servir de estímulo para futuras iniciativas no sentido de adotar novas abordagens pedagógicas no ensino superior em Administração no Brasil e de aprimorar o ferramental tecnológico disponível.

Foram consideráveis os esforços até se chegar ao ambiente para aprendizagem em gestão de projetos apresentado. Parte importante destes esforços foram investidos no desenvolvimento do sistema E-BIACS, especialmente no projeto da arquitetura do sistema e na programação das funções de recomendação. A construção do modelo de conhecimentos exigiu diversas reuniões de trabalho com o professor participante para análise, síntese e ajustes no modelo de conhecimentos. O sistema E-BIACS e o modelo de conhecimentos em gestão de projetos construído são contribuições concretas desta pesquisa. Novas aplicações, que abordem outros domínios, podem ajudar a avalidar a flexibilidade da arquitetura do sistema para diferentes disciplinas. O modelo de conhecimentos em gestão de projetos proposto pode servir de ponto de partida para a construção de novos ambientes de aprendizagem em gestão de projetos no E-BIACS, confirmando a flexibilidade da arquitetura do sistema para a criação de variadas perspectivas sobre os conteúdos de uma disciplina.

\section{REFERÊNCIAS BIBLIOGRÁFICAS}

ACHTENHAGEN, F. Criteria for the development of complex teaching-learning environments. Instructional Science, v. 29, p. 361-380, 2001.

ADDA, M.; VALTCHEV, P.; MISSAOUI, R.; DJERABA, C. Toward Recommendation Based on Ontology- Powered Web-Usage Mining. IEEE, Internet Computing, v. 11, n. 4, p. 45-52, 2007.

BARROWS, H. S. Problem-based learning in medicine and beyond: A brief overview. In W. H. Gijselaers (Ed.), New directions for teaching and learning, San Francisco: Jossey-Bass, v. 1996, n. 68, p. 3-11, 1996.

CHECKLAND, P. Systems Thinking, Systems Practice. John Wiley, Chinchester, 1981.

FERREIRA-SATLER, M.; ROMERO, F.; MENENDEZ-DOMINGUEZ, V.; ZAPATA, A.; PRIETO, M. Fuzzy ontologies-based user profiles applied to enhance e-learning activities. Springer Soft Comput. v. 16, p. 1129-1141, 2012.

FRÓES, M. F.; SANTOS, E. R. Questionário aplicado na pesquisa. Disponível em:

$<$ https://www.dropbox.com/s/kcqpook34ynsn2l/question\%C3\%A1rio_v1.pdf $>$. Acesso em: 28 jun. 2014.

GIJBELS, D.; VAN DE WATERING, G.; DOCHY, F.; VAN DEN BOSSCHE, P. New learning environments and constructivism: The students' perspective. Instructional Science, v. 34, p. 213-226, 2006.

JONASSEN, D. H.; IONAS, I. G. Designing effective supports for causal reasoning. Education Tech Research Dev. n. 56, p. 287-308, 2008.

KANELLOPOULOS, D.; KOTSIANTIS, S.; PINTELAS, P. Ontology-Based Learning Applications: a development methodology. Procedings of the $24^{\text {th }}$ IASTED International Multi-Conference of Software Engineering, 2006. 
KLOPPENBORG, T.; BAUCUS, S. Project Management in Local Nonprofit Organizations: Engaging Students in Problem-Based Learning. Journal of Management Education, v. 28, n. 5, p. 610-629, 2004.

LIAO, S.; KAO, K.; LIAO, I.; CHEN, H.; HUANG, S. PORE: a personal ontology recommender system for digital libraries. The Electronic Library. v. 27, n. 3, p. 496-508, 2009.

LÓPEZ-NORES, M.; BLANCO-FERNÁNDEZ, Y.; PAZOS-ARIAS, J. J.; GILSOLLA, A. Property-based collaborative filtering for health-aware recommender systems. Expert Systems with Applications, v.39, p. 7451-7457, 2012.

LOYENS, S. M. M.; MAGDA, J.; RIKERS, R. Self-Directed Learning in ProblemBased Learning and its Relationships with Self-Regulated Learning. Educ Psychol Rev, v. 20, p. 411-427, 2008.

MAIDEL, V.; SHOVAL, P.; SHAPIRA, B.; TAIEB-MAIMON, M. Ontological content-based filtering for personalized newspapers: A method and its evaluation. Online Information Review. v. 34, n. 5, p. 729-756, 2010.

MENEZES, C. S.; VALE, K. S. B.; CRUZ, P.O. AICAPA - Um Ambiente Inteligente e Colaborativo para Apoio à Produção Acadêmica. RENOTE. Revista Novas Tecnologias na Educação, v. 11, n. 3, 2013.

MONDADORI, M. G.; SANTOS, E. R. Uma proposta de princípios para a construção de ambientes de aprendizagem com orientação construtivista para o ensino em administração. RENOTE. Revista Novas Tecnologias na Educação, v. 4, p. 1-11, 2006.

PELLEGRINO, J. Complex learning environments: Connecting learning theory, Instructional Design, and Technology. In: SEEL, N.; DIJKSTRA, S. Curriculum, Plans, and Process in Instructional Design - International Perspectives. Lawrence Erlbaum Associates, 2004. p. 25-49.

PEREIRA, F. R.; RIGO, S. J. Utilização de processamento de linguagem natural e ontologias na análise qualitativa de frases curtas. RENOTE. Revista Novas Tecnologias na Educação, v. 11, n. 2, 2013.

SALES, A. B.; DEL, A. M.; SALES, M. B. Avaliação da Aplicação da Aprendizagem Baseada em Problemas na Disciplina de Interação Humano e Computador de curso de Engenharia de Software. RENOTE. Revista Novas Tecnologias na Educação, v. 11, n. 3, 2013.

SANTOS, E.R. BIACS - Base Inteligente para Aquisição de Conhecimentos de Sistemas. Instituto Nacional da Propriedade Industrial. Rio de Janeiro, 1989.

SEEL, N.; DIJKSTRA, S. Curriculum, Plans, and Processes in Instructional Design - International Perspectives, London: Lawrence Erlbaum Associates, 2004. 385 p.

SHERWOOD, A. Problem-Based Learning in Management Education: A Framework for Designing Context. Journal of Management Education. v. 28, n. 5, p. 536-557, 2004. 